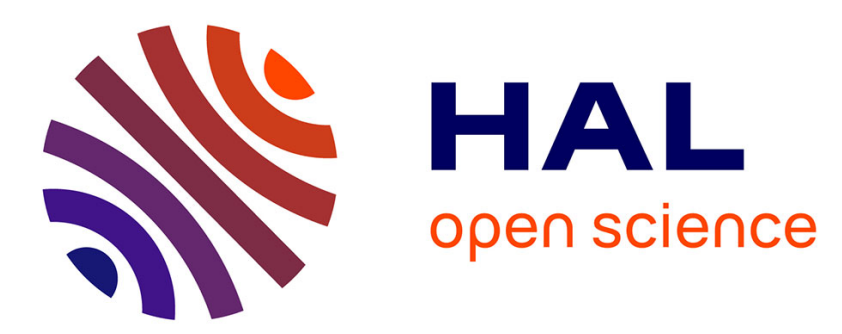

\title{
Direction of arrival estimation by modified orthogonal propagator method with linear prediction in low SNR scenarios
}

Meng Sun, Yide Wang, Jingjing Pan

\section{- To cite this version:}

Meng Sun, Yide Wang, Jingjing Pan. Direction of arrival estimation by modified orthogonal propagator method with linear prediction in low SNR scenarios. Signal Processing, 2019, 156, pp.41-45. 10.1016/j.sigpro.2018.10.013 . hal-01908223

\section{HAL Id: hal-01908223 \\ https://hal.science/hal-01908223}

Submitted on 9 Nov 2018

HAL is a multi-disciplinary open access archive for the deposit and dissemination of scientific research documents, whether they are published or not. The documents may come from teaching and research institutions in France or abroad, or from public or private research centers.
L'archive ouverte pluridisciplinaire HAL, est destinée au dépôt et à la diffusion de documents scientifiques de niveau recherche, publiés ou non, émanant des établissements d'enseignement et de recherche français ou étrangers, des laboratoires publics ou privés. 


\title{
Direction of arrival estimation by modified orthogonal propagator method with linear prediction in low SNR scenarios
}

\author{
Meng Sun ${ }^{\mathrm{a}}$, Yide Wang ${ }^{\mathrm{b}}$, Jingjing Pan ${ }^{\mathrm{b}}$ \\ ${ }^{a}$ Information Engineering College, Shanghai Maritime University, Shanghai, People's Republic of \\ China \\ ${ }^{b}$ Institut d' Electronique et Télécommunications de Rennes (IETR), Université de Nantes, UMR \\ CNRS 6164, Rue Christian Pauc BP 50609, Nantes 44306, France \\ Email:(jingjing.pan1@etu.univ-nantes.fr)
}

\section{Abstract}

In propagator based methods for Direction of Arrival (DoA) finding, conventional approaches for eliminating the noise impact are based on signal enhancement or noise power estimation. In this paper, a new method is introduced from another point of view. When the noise is an additive spatially and temporally white Gaussian noise, theoretically, it only impacts the diagonal elements of the data covariance matrix. Firstly, the proposed method utilizes the principle of Linear Prediction (LP) to rebuild the denoised diagonal elements of the data covariance matrix. Then, the Orthogonal Propagator Method (OPM) is directly applied for DoA estimation. Compared with the conventional OPM-based methods, the proposed method is more robust to the noise, especially in low Signal to Noise Ratio (SNR) scenarios. Simulation examples are provided to demonstrate the performance of the proposed method. Keywords: Direction of Arrival (DoA) estimation, Linear Prediction (LP), Orthogonal Propagator Method (OPM). 


\section{Introduction}

Direction of Arrival (DoA) estimation is of practical importance in many engineering applications, for example, radar, sonar, and wireless communications $[1,2]$. A variety of methods have been proposed to estimate DoA. MUSIC [3] and ESPRIT 5 [4] have asymptotically infinite resolution but high computational burden, since they are based on the EigenValue Decomposition (EVD) of the data covariance matrix. Propagator-based methods, for example, Propagator Method (PM) $[5,6]$ and Orthogonal Propagator Method (OPM) [7], have high resolution and accuracy by using only linear operations without any EVD operation, which make real time processing possible.

Nevertheless, propagator-based methods are developed with signal models under ideal condition and without the consideration of noise. Thus, their performance is degraded in low SNR scenarios. There are three possible solutions to solve this problem. Firstly, the least squares method can be applied to estimate the propagator (the principle of PM and OPM). However, this method only reduces the noise impact but does not eliminate it [5]. The other two possible solutions are either to estimate the power of the noise $[8,9]$ or to apply signal enhancement techniques [10]. In [10], Li et al. propose to enhance the received signals by using Karhunen-Loève Transformation (KLT). Stoica et al. [8] propose an eigenvalue-based method to estimate the power of the noise. However, these two methods require EVD. In addition, Marcos and Sanchez-Araujo [6] and Stoica et al. [8] also propose two different linear operation methods for estimating the power of the noise without EVD.

In this paper, we propose a new way to eliminate the noise impact. When the 


\section{Signal model}

Consider $K$ far-field narrow-band signals impinging on a ULA with $N$ isotropic antenna elements, as shown in Fig. 1. The received signals are supposed totally uncorrelated. The indexes of the antennas are set to $0,1, \ldots, N-1$. Then, the receiyed signal at the $m$ th element can be written as

$$
r_{m}(t)=\sum_{k=1}^{K} s_{k}(t) \exp \left(j 2 m \pi \frac{d}{\lambda} \sin \theta_{k}\right)+n_{m}(t)
$$


where $s_{k}(t)$ is the complex envelope of the $k$ th signal, with DOA $\theta_{k}$, received at the 0th element; $n_{m}(t)$ is an additive spatially and temporally white Gaussian noise at the $m$ th element with zero mean and variance $\sigma^{2} ; d$ denotes the distance between two adjacent elements and $\lambda$ is the wavelength of the received signals. (1) can be written in the following vector form:

$$
\mathbf{r}(t)=\mathbf{A s}(t)+\mathbf{n}(t)
$$

with the following notation definitions: $\mathbf{r}(t)=\left[r_{0}(t) r_{1}(t) \cdots r_{N-1}(t)\right]^{T}$ is the $(N \times$ 1) received signal vector, the superscript $T$ denotes the transpose operation; $\mathbf{A}=$ $\left[\mathbf{a}\left(\theta_{1}\right) \mathbf{a}\left(\theta_{2}\right) \ldots \mathbf{a}\left(\theta_{K}\right)\right]$ is the $(N \times K)$ directional matrix; $\mathbf{a}\left(\theta_{k}\right)=\left[1 \exp \left(j 2 \pi \frac{d}{\lambda} \sin \theta_{k}\right)\right.$ $\left.\ldots \exp \left(j 2(N-1) \pi \frac{d}{\lambda} \sin \theta_{k}\right)\right]^{T}$ is the directional vector; $\mathbf{s}(t)=\left[\begin{array}{llll}s_{1}(t) & s_{2}(t) & \cdots & s_{K}(t)\end{array}\right]^{T}$ is the $(K \times 1)$ source vector; $\mathbf{n}(t)=\left[n_{0}(t) n_{1}(t) \cdots n_{N-1}(t)\right]^{T}$ is the $(N \times 1)$ noise vector, with zero mean and covariance matrix $\sigma^{2} \mathbf{I}, \mathbf{I}$ is the $(N \times N)$ identity matrix.

According to signal model (2) and assuming that the noise is independent from the signals, the covariance matrix $\mathbf{R}$ can be written as

$$
\widehat{\mathbf{R}}=E\left\{\mathbf{r}(t) \mathbf{r}^{H}(t)\right\}=\mathbf{A} \Gamma \mathbf{A}^{H}+\sigma^{2} \mathbf{I}
$$

where $E\{:\}$ denotes the expectation, $\boldsymbol{\Gamma}=E\left\{\mathbf{s}(t) \mathbf{s}^{H}(t)\right\}$ is the $(K \times K)$ dimensional 
jugate transpose operation. Therefore, the elements of $\mathbf{R}$ can be expressed as

$$
R(m-n)=\mathbf{R}(m, n)= \begin{cases}\sum_{k=1}^{K} P_{s_{k}}+\sigma^{2} & m=n \\ \sum_{k=1}^{K} P_{s_{k}} \exp \left(j 2 \pi(m-n) \frac{d}{\lambda} \sin \theta_{k}\right) & m \neq n\end{cases}
$$

where $m, n \in[1, \ldots N], P_{s_{k}}$ is the power of the $k$ th signal. As shown in (4), the noise is presented only in the diagonal elements of $\mathbf{R}$, which can be rewritten as

$$
\mathbf{R}=\left(\begin{array}{cccc}
R(0) & R(-1) & \ldots & R(1-N) \\
R(1) & R(0) & \ldots & R(2-N) \\
\ldots & \ldots & \ldots & \ldots \\
R(N-1) & R(N-2) & \ldots & R(0)
\end{array}\right)
$$

\section{Modified OPM (OPM-LP) for DoA estimation}

The objective of this work is to build a denoised covariance matrix for the implementation of OPM. Since $\mathbf{R}$ is a Toeplitz and Hermitian matrix, the main diagonal elements are the same (equal to $R(0)$ ). According to (4), the elements in $\mathbf{R}$ are a linear combination of exponentials. Therefore, we can use LP for restoring $R(0)$. A Toeplitz and Hermitian matrix is uniquely determined by its first line (column). In the proposed method, the information of the first column is used in the estimation of $R(0)$. The first column of $\mathbf{R}$ is partitioned into several overlapping sub-sequences and each sub-sequence contains $L$ elements, as shown in Fig. 2. The LP prediction 
$\hat{R}(n)$ for $R(n)$ has the following form:

$$
\hat{R}(n)=\sum_{l=1}^{L} \omega_{l} R(n+l), n=0, \ldots, N-L-1
$$

where $\omega_{l}(l=1, \ldots, L)$ is the prediction coefficient. Therefore, we can obtain the denoised element $R(0)$ from the rest of the elements of the first column with the predictions coefficients. The prediction coefficients can be estimated using the subsequences $\mathbf{r}_{2}, \mathbf{r}_{3}, \ldots \mathbf{r}_{N-L}$. In this case, the LP expression can be formulated as

$$
\underbrace{\left[\begin{array}{cccc}
R(2) & R(3) & \ldots & R(L+1) \\
\vdots & \vdots & & \vdots \\
R(N-L) & R(N-L+1) & \ldots & R(N-1)
\end{array}\right]}_{\mathbf{x}} \underbrace{\left[\begin{array}{c}
\omega_{1} \\
\vdots \\
\omega_{L}
\end{array}\right]}_{\boldsymbol{\omega}}=\underbrace{\left[\begin{array}{c}
R(1) \\
\vdots \\
R(N-L-1)
\end{array}\right]}_{\mathbf{y}} .
$$

Since $\mathbf{X}$ and $\mathbf{y}$ are known, the coefficient vector $\boldsymbol{\omega}$ can be estimated by the leastsquares approach:

$$
\boldsymbol{\omega}=\left(\mathbf{X}^{H} \mathbf{X}\right)^{-1} \mathbf{X}^{H} \mathbf{y}=\mathbf{X}^{+} \mathbf{y}
$$

where $\mathbf{X}^{+}$is the Moore-Penrose inverse of $\mathbf{X}$. One should notice that $\boldsymbol{\omega}$ has a unique solution only when $N-L-1 \geq L$. Therefore, in order to resolve $K$ sources, the constraint for $L$ is $K \leq L \leq \frac{1}{2}(N-1)$. Then, we can calculate $\hat{R}(0)$ with the estimated $\boldsymbol{\omega}$ :

$$
\hat{R}(0)=\sum_{l=1}^{L} \omega_{l} R(l)
$$

Since the noise is removed, OPM [7] can be directly applied on the denoised data covariance matrix for DoA estimation. 


\section{Simulations and discussion}

In this section, the performance of OPM-LP is tested on 4 different numerical simulations. A ULA with 15 isotropic elements is considered, along with two far-field uncorrelated narrow-band signals with equal power, that is $N=15, K=2$. The number of elements in each sub-sequence $L$ is equal to 6 . The distance between two adjacent elements is half of the wavelength.

In the first experiment, the pseudo-spectrums of the proposed method, conventional OPM and OPM-MPEN are estimated. Two cases are taken into account with different DoAs of the incoming signals: Case $a$. $\theta_{1} \neq-5^{\circ}$ and $\theta_{2}=4^{\circ}$; Case $b$. $\theta_{1}=-1^{\circ}$ and $\theta_{2}=2^{\circ}$. SNR is fixed at $-5 \mathrm{~dB}$. The data covariance matrix is estimated from 1000 independent snapshots. The pseudo-spectrum search is performed over $\left[-20^{\circ}, 20^{\circ}\right]$ with step size $0.001^{\circ}$. Figs. 3 and 4 show the pseudo-spectrums of the proposed method and the conventional OPM and OPM-MPEN for Cases $a$ and $b$, respectively. For Case $a$, both the proposed method and OPM-MPEN can detect the true DOAs of the incoming signals, as shown in Fig. 3. The result of the conventional OPM is biassed. When the angle separation becomes small, in Case $b$, the conventional OPM fails to estimate the DoAs while OPM-MPEN estimates the DoA with bias. However, the proposed method remains accurate, even when the angle separation is small.

In the second simulation, the performance of the proposed method versus SNR is assessed with a Monte-Carlo process of 500 independent runs. The Root Mean 
Square Error (RMSE) of the estimated DoA is defined as follows:

$$
\mathrm{RMSE}=\sqrt{\frac{1}{K J} \sum_{j=1}^{J} \sum_{k=1}^{K}\left(\hat{\theta}_{k j}-\theta_{k}\right)^{2}}
$$

where $\hat{\theta}_{k j}$ denotes the estimated DoA of the $k$ th incoming signal for the $j$ th rum, and $\theta_{k}$ the true value. SNR varies from $-10 \mathrm{~dB}$ to $5 \mathrm{~dB}$. The angles in Case $b$ are applied here. The proposed method is compared with OPM-MPEN, OPM-EVD [8], OPM-KLT [10], and also Cramér-Rao Bound (CRB). Fig. 5 plots the RMSE of DoA estimation with respect to SNR. For all the compared methods, the RMSE is continuously decreasing when SNR increases. It can be seen from Fig. 5 that the RMSE of the proposed method is smaller than that of OPM-MPEN and OPMEVD, especially in low SNR scenarios. Although OPM-KLT has smaller RMSE than the proposed method at lower SNR, the proposed method has a more significant decrease of RMSE as SNR increases. Moreover, the computational complexity of the proposed denoising procedure $(\mathrm{LP})$ is $O\left(L^{2}(N-L-1)\right)$, which is similar to MPEN $\left(O\left(N^{2}(K+1)+N K^{2}\right)\right)$ and is much smaller than that of EVD and $\operatorname{KLT}\left(O\left(N^{3}\right)\right)$.

In the third simulation, the statistical performance of the proposed method versus the angle separation between two incoming signals is assessed with 500 independent runs of the algorithm. One of the incoming signals is fixed at $\theta_{1}=0^{\circ}$ while the other comes from $\theta_{2}=\theta_{1}+\Delta \theta$. SNR is fixed at $-5 \mathrm{~dB}$ and $\Delta \theta$ varies from $1^{\circ}$ to $10^{\circ}$. As shown in Fig. 6, when the angle separation is small $\left(1^{\circ}\right.$ to $\left.2^{\circ}\right)$, all methods fail to detect the true DoAs of the incoming signals. Moreover, the RMSE continuously decreases when the angle separation increases. Similar to the second simulation, the proposed method offers better performance than that of OPM-MPEN, OPM-KLT 
and OPM-EVD.

In the last simulation, the performance of the proposed method versus the length of sub-sequence $L$ is studied with 500 independent runs. The angles in Case $b$ are applied, SNR $=-5$ dB. $L \in[2,7]$ due to $K \leq L \leq \frac{1}{2}(N-1)$. It can be seen from Fig. 7, when $L=5$, the RMSE reaches its minimum. Therefore, in this situation, $L=5$ is optimal value for DoA estimation, however, the RMSE shows similar value with different $L$.

\section{Conclusion}

This paper proposes a new method OPM-LP for DoA estimation in low SNR scenarios. This new technique utilizes the principle of LP, which offers a new way to eliminate the noise impact. Simulation results show the stability, robustness and efficiency of the proposed method. In perspective, the proposed method will be tested with the real measurement.

\section{References}

[1] H. Krim, M. Viberg, Two decades of array signal processing research: the parametric approach, IEEE signal processing magazine 13 (4) (1996) 67-94.

[2] T. E. Tuncer, B. Friedlander, Practical Aspects of Design and Application of Direction-Finding Systems, in: Classical and Modern Direction-of-Arrival Estimation, Elsevier, (2009) 53-92.

[3] R. O. Schmidt, Multiple emitter location and signal parameter estimation, IEEE Transactions on Antennas and Propagation , 34 (3) (1986) 276-280. 
[4] R. Roy, T. Kailath, ESPRIT-estimation of signal parameters via rotational invariance techniques, IEEE Transactions on Acoustics, Speech and Signal Processing, 37 (7) (1989) 984-995.

[5] J. Munier, G. Y. Delisle, Spatial analysis using new properties of the crossspectral matrix, IEEE Transactions on Signal Processing 39 (3) (1991) 746-749.

[6] S. Marcos, J. Sanchez-Araujo, Méthodes Linéaires Haute Résolution pour l'Estimation de Directions D'Arrivée de Sources. Performances Asymptotiques et Complexité, Traitement du signal 14 (2) (1997) 99-116.

[7] S. Marcos, A. Marsal, M. Benidir, The propagator method for source bearing estimation, Signal processing 42 (2) (1995) 121-138.

[8] P. Stoica, T. Söderström, V. Šimonyt, On estimating the noise power in array processing, Signal Processing 26 (2) (1992) 205-220.

[9] A. Mariani, A. Giorgetti, M. Chiani, Effects of noise power estimation on energy detection for cognitive radio applications, IEEE Transactions on Communications 59 (12) (2011) $3410-3420$.

[10] J. Li, C. Le Bastard, Y. Wang, G. Wei, B. Ma, M. Sun, Enhanced GPR signal for layered media time-delay estimation in low-SNR scenario, IEEE Geoscience and Remote Sensing Letters 13 (3) (2016) 299-303.

[11] S. L. Marple, S. L. Marple, Digital spectral analysis: with applications, vol. 5, Prentice-Hall Englewood Cliffs, NJ, 1987. 


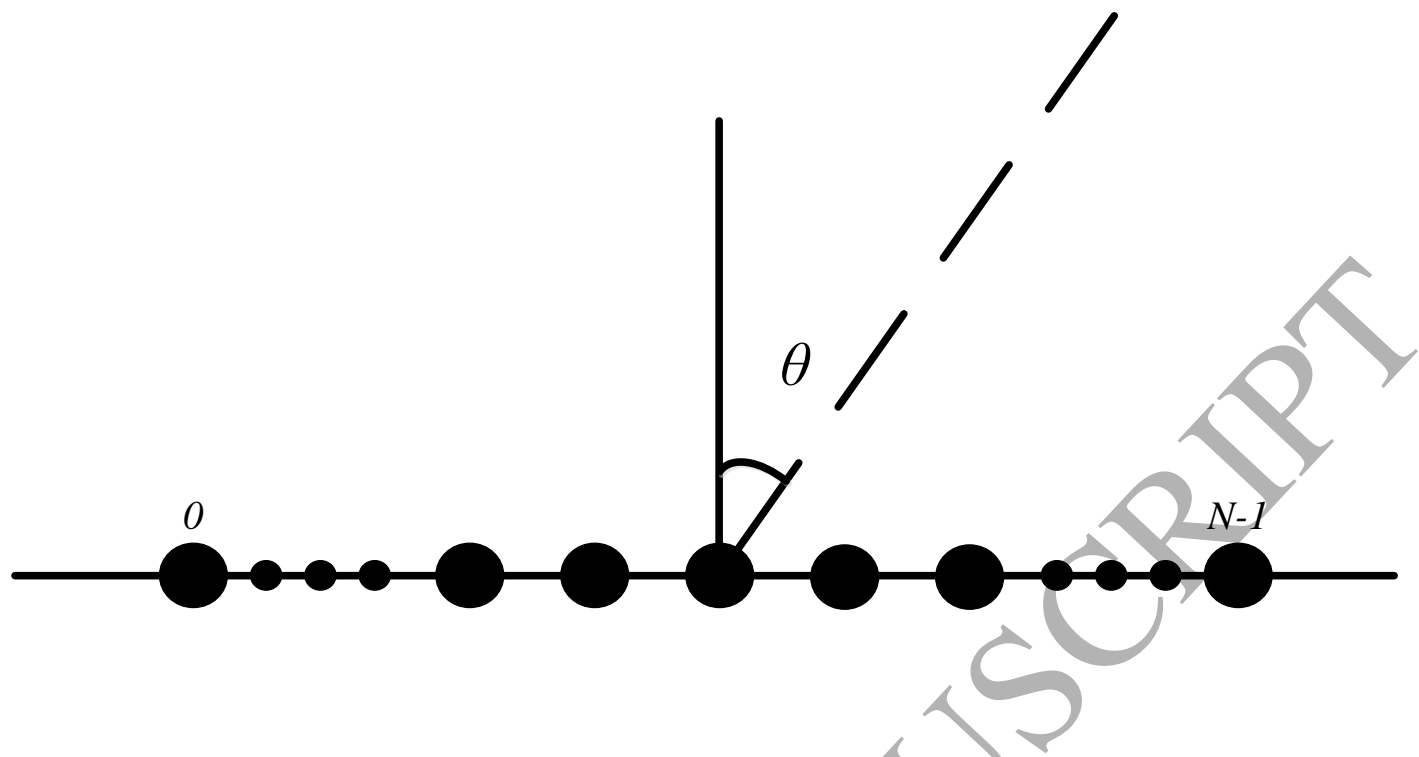

Figure 1: Uniform Linear Array Configuration

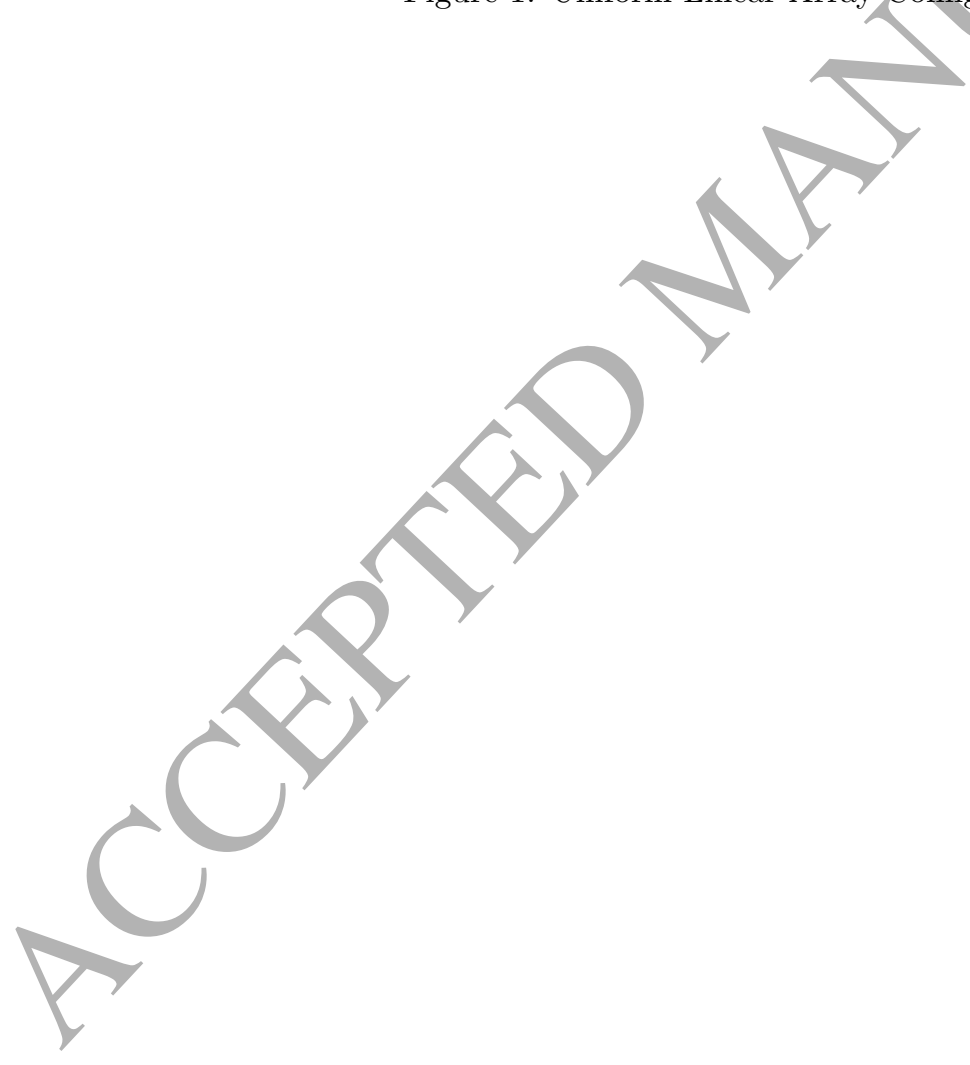




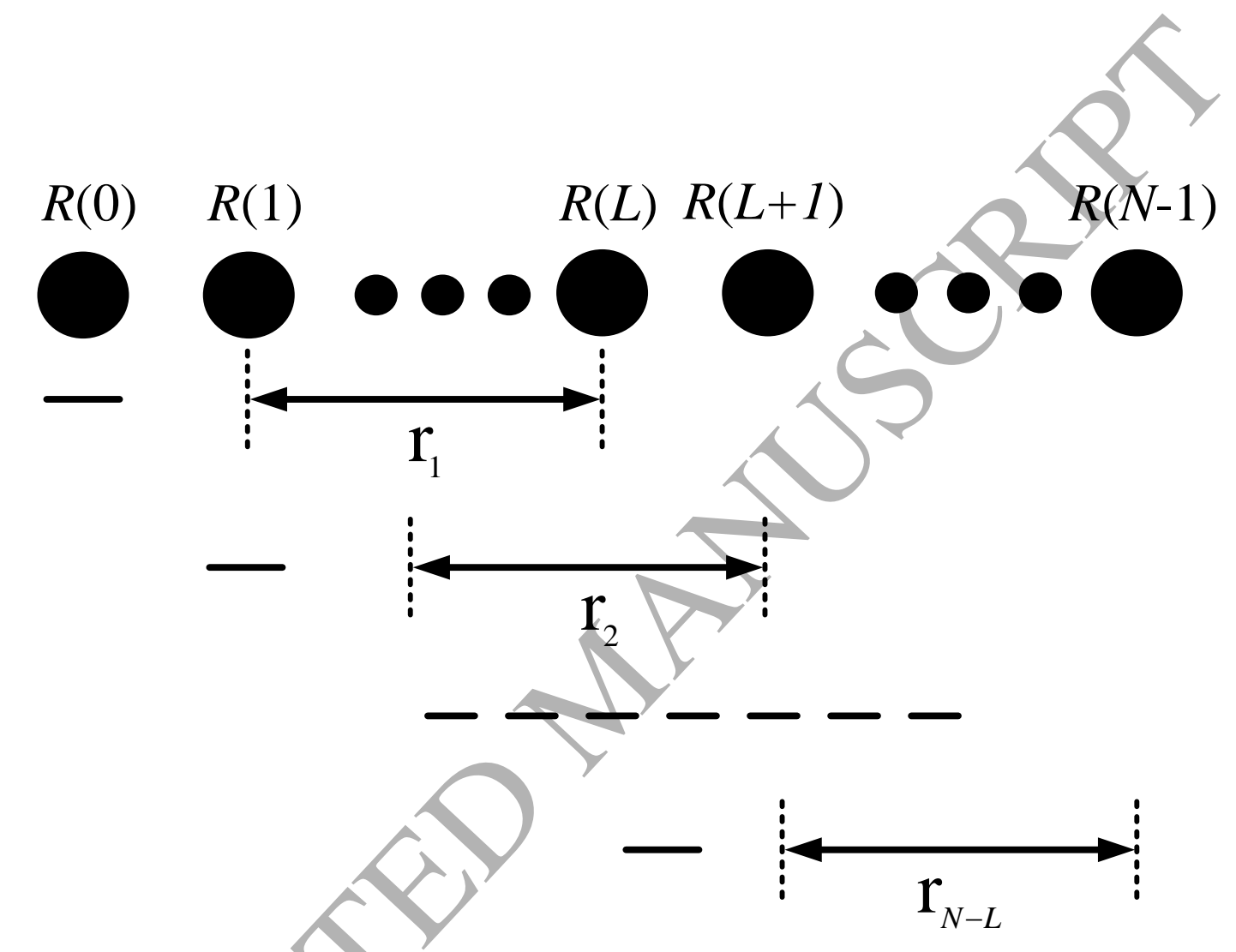

Figure 2: Set of the overlapping sub-sequences of the first line of $\mathbf{R}$

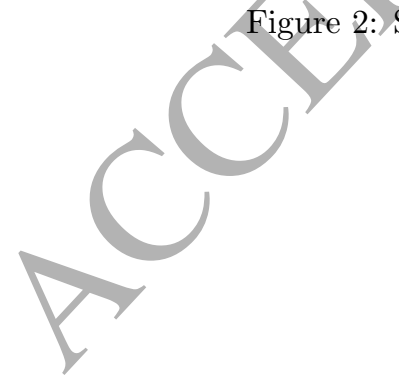




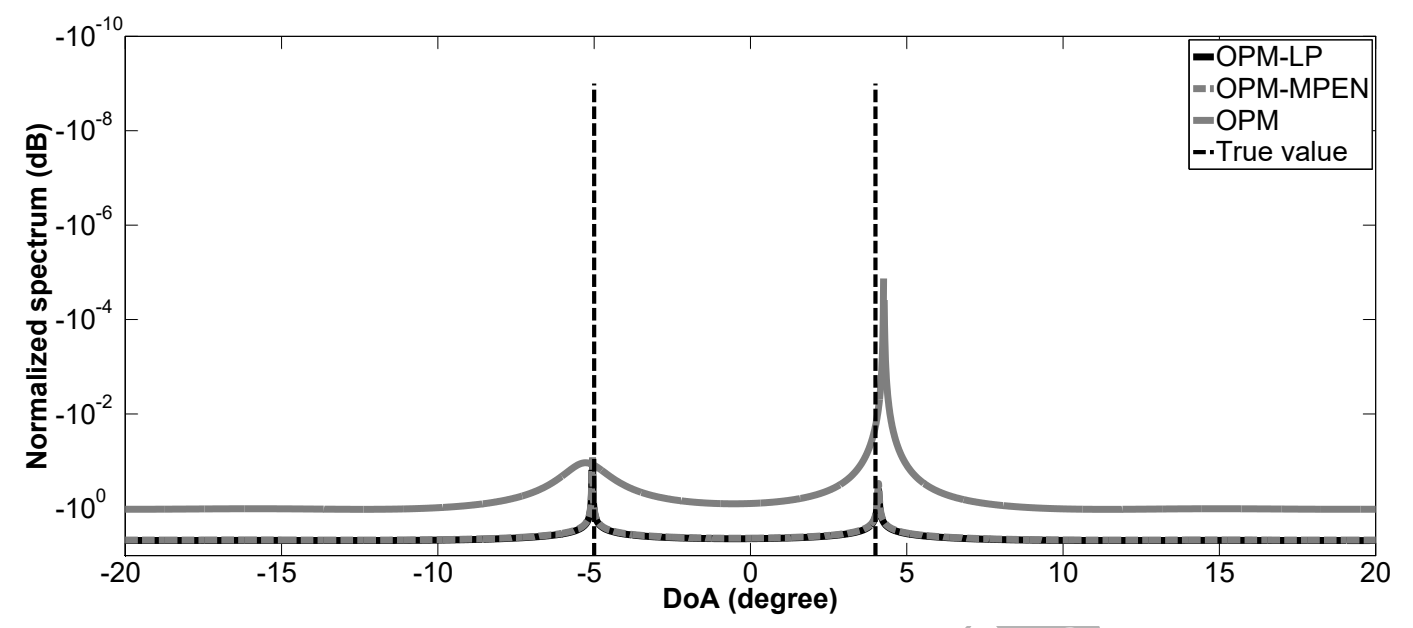

Figure 3: Case $a$, pseudo-spectrums of the OPM-LP, conventional OPM and OPM-MPEN for DoA estimation, the two DoAs are $\theta_{1}=-5^{\circ}$ and $\theta_{2}=4^{\circ}$.

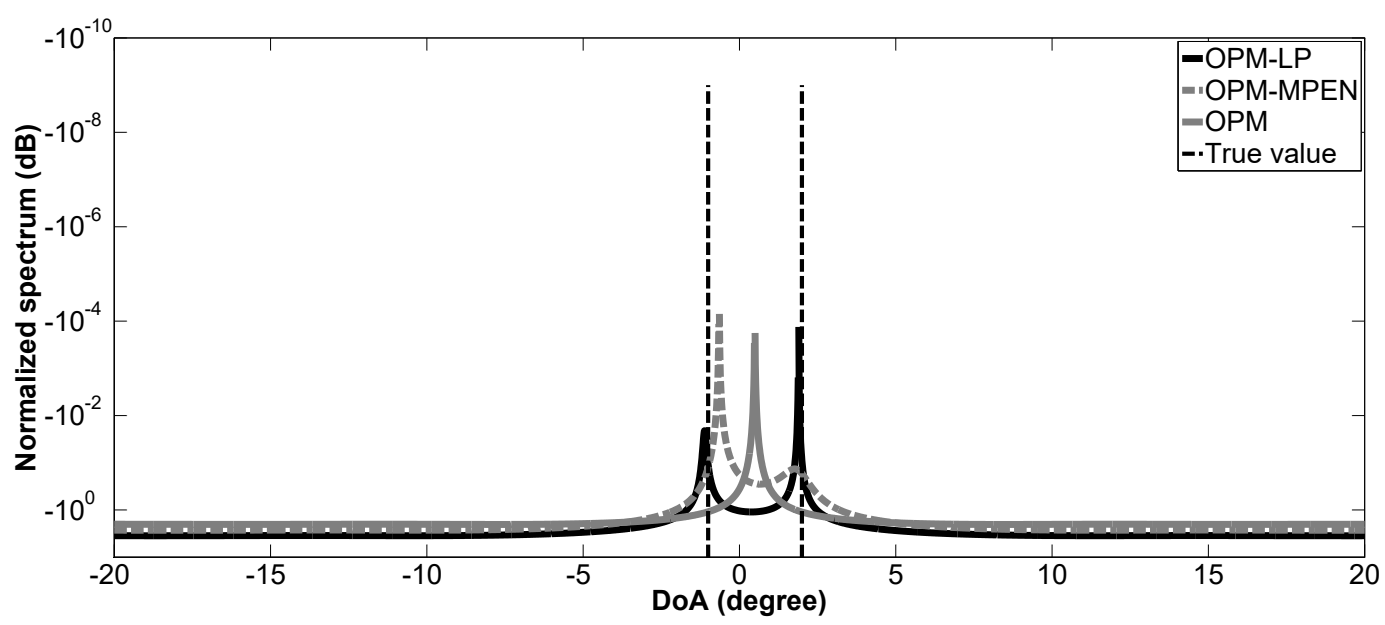

Figure 4: Case $b$, pseudo-spectrums of the OPM-LP, conventional OPM and OPM-MPEN for DoA estimation, the two DoAs are $\theta_{1}=-1^{\circ}$ and $\theta_{2}=2^{\circ}$. 


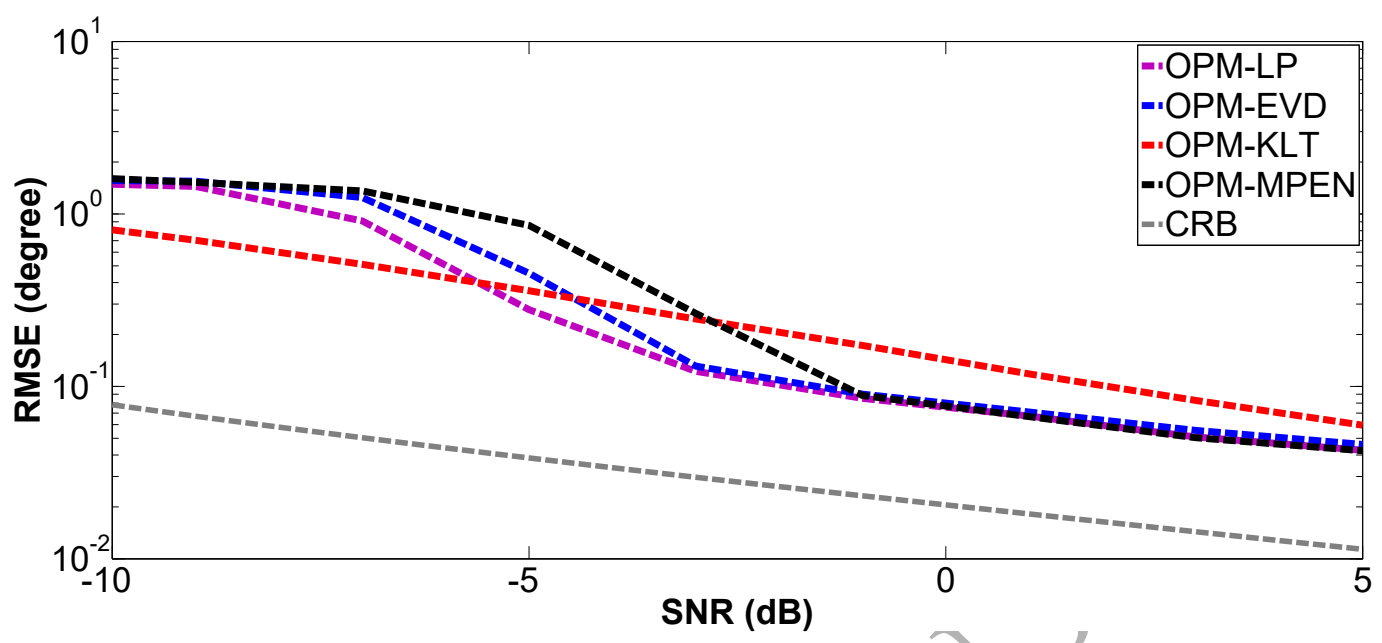

Figure 5: Case b, RMSE of DoA estimation versus SNR

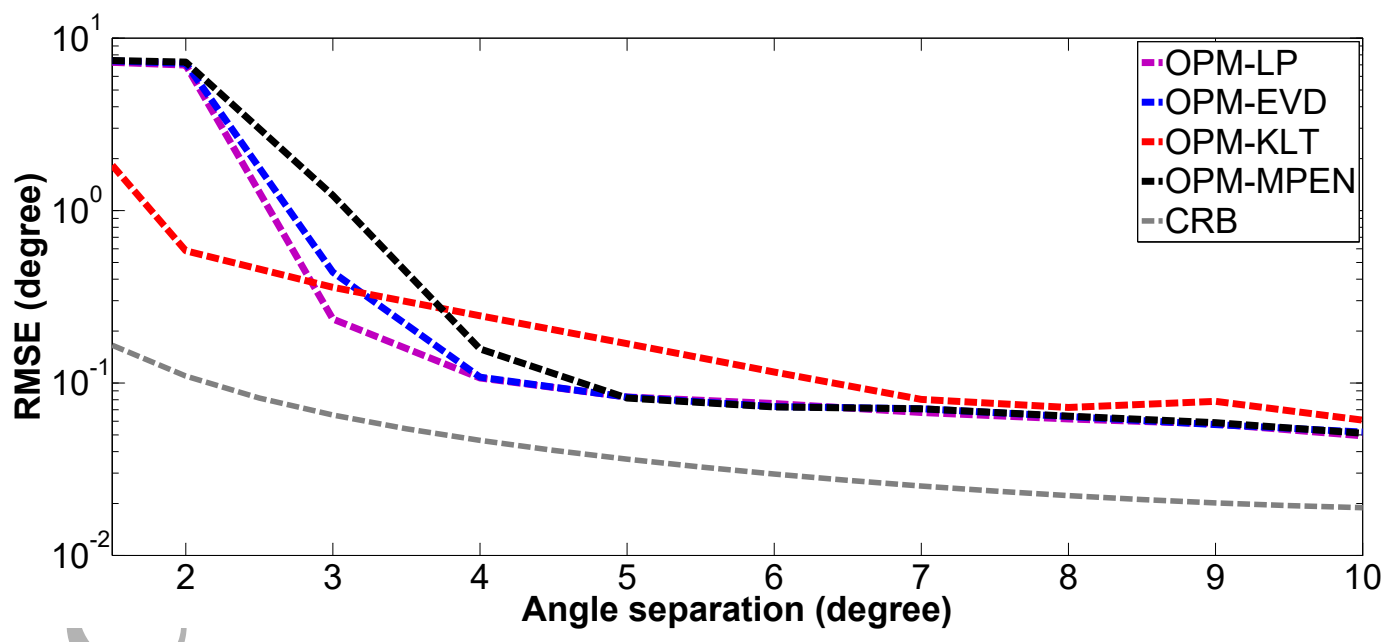

Figure 6: RMSE of DoA estimation in terms of angle separation 


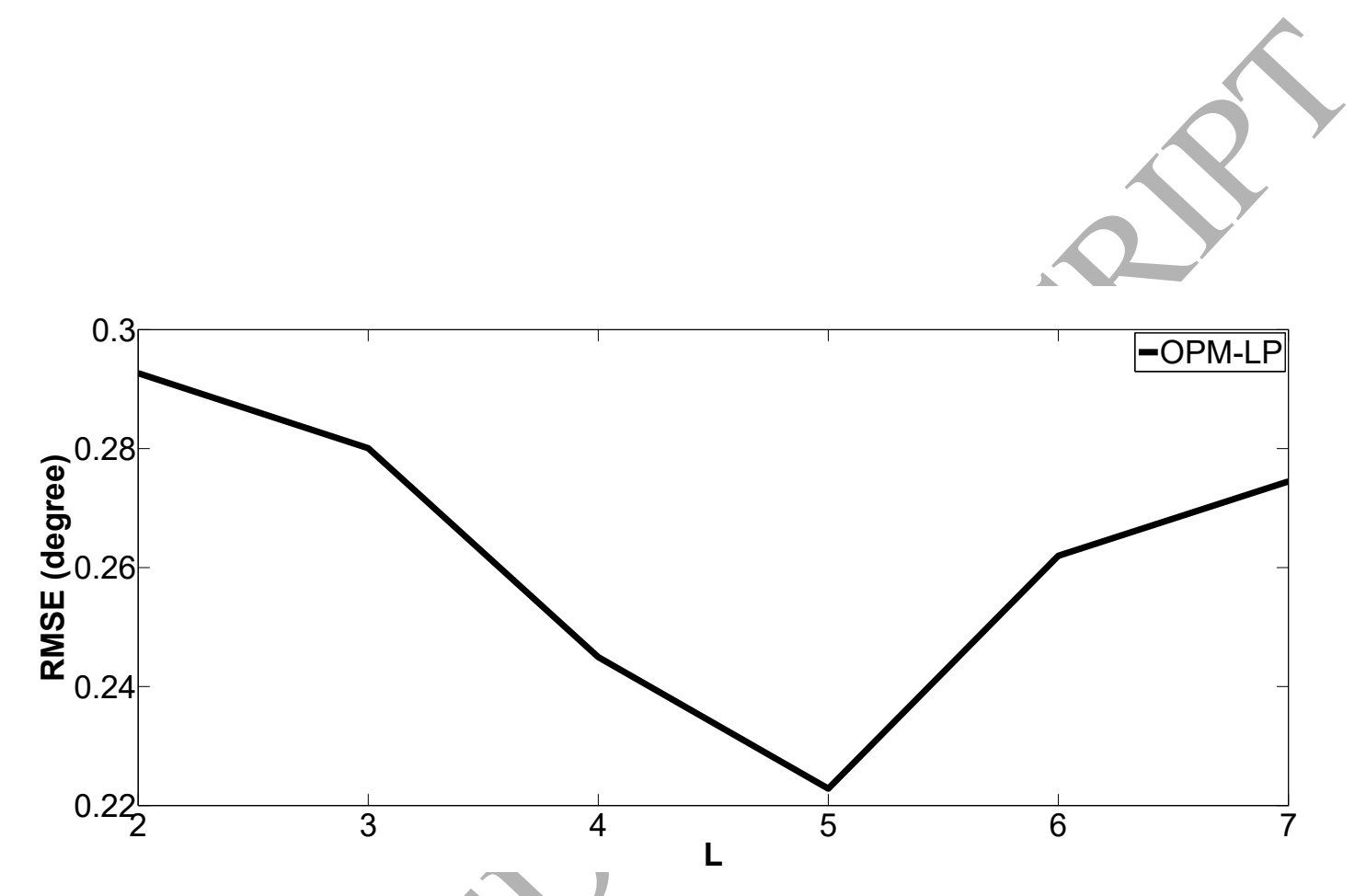

Figure 7: RMSE of DoA estimation as a function of $L$

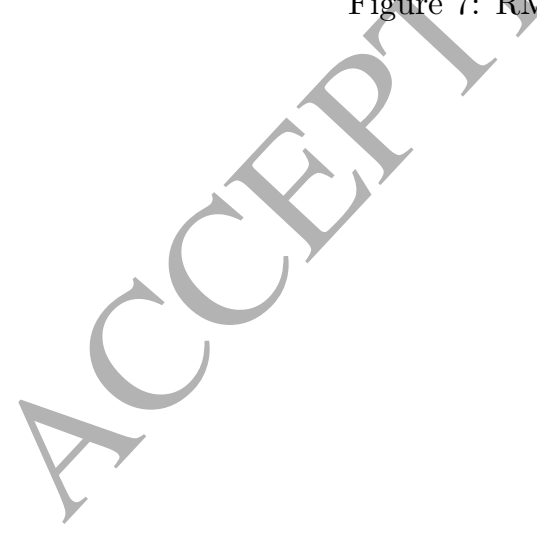

